\title{
Sensitivity to serine starvation
}

The transcription factor and tumour suppressor p53 (encoded by TP53) is known to regulate several different cellular pathways that include a number of metabolic processes. p53 is required for the survival of cells in the absence of glucose, so Karen Vousden and colleagues investigated the response of p53 wild-type (TP53 $\left.{ }^{+/+}\right)$ and p53-null (TP53 ${ }^{--}$) human cancer cell lines to the loss of other nutrients. Although TP53 ${ }^{+/+}$cells showed reduced rates of proliferation in the absence of serine and glycine, TP53 $3^{-/-}$ cells had even fewer proliferating cells and a loss of viability. Moreover, TP53 ${ }^{-/}$HCT116 xenografts in mice fed a serine- and glycine-free diet had a significantly reduced tumour volume compared with p53 wild-type HCT116 xenografts, the growth of which was also compromised in these animals. Loss of serine rather than

Both wild-type and p53-null cancer cells responded to serine loss by switching on the serine synthesis pathway (SSP)
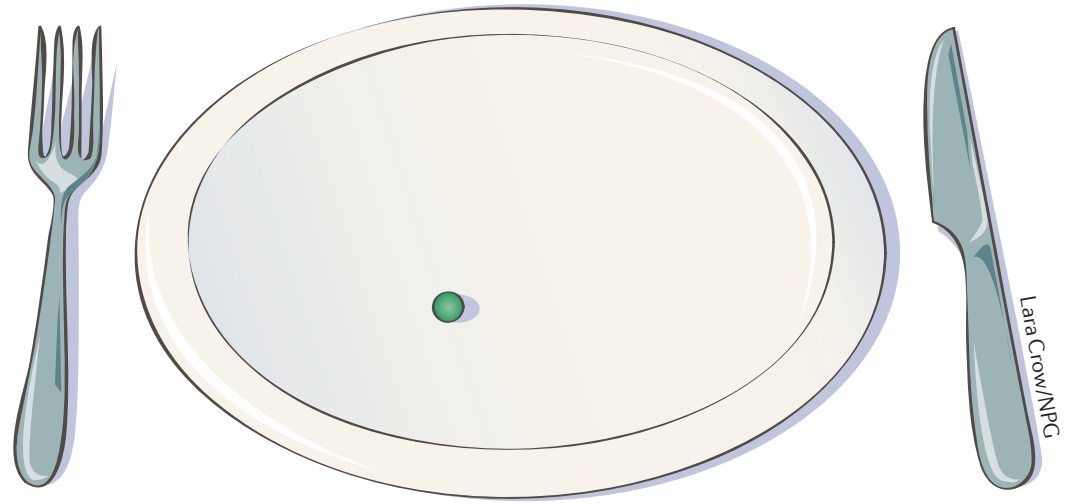

responded to serine starvation by increasing metabolic flux through the TCA cycle, this was reduced over time in the wild-type cells but was maintained in the p53-null cells (shown by increased oxygen consumption). The authors also found that expression of the p53-regulated cyclin-dependent kinase inhibitor p21 was induced by serine starvation through a subtle increase in p53 expression, resulting in cell cycle arrest. Cell cycle arrest was substantially reduced in the p53-null cells and in cells in which p21 expression was knocked down. The capacity to produce reduced glutathione (GSH) in the absence of serine was also compromised in p53-null cells, and this, combined with increased oxygen consumption, increased the levels of reactive oxygen species. Indeed, loss of proliferation of p53-null cells in the absence of serine could be rescued by treatment with an anti-oxidant (such as GSH) and pyruvate (to increase flux through the TCA cycle).

Thus, these results indicate that p53 is required for cells to alter their metabolism in the absence of serine and that the induction of a cell cycle arrest facilitates this adaptive response. They also show that serine is required to sustain aerobic glycolysis (the Warburg effect) in cancer cells, and as such the effects of reducing the level of serine available to cancer cells are worthy of further investigation.

Nicola McCarthy

ORIGINAL RESEARCH PAPER Maddocks, O. D. K et al. Serine starvation induces stress and p53dependent metabolic remodelling in cancer cells. Nature 16 Dec 2012 (doi:10.1038/nature11743) 